\title{
Aberrant Activation of the Hedgehog Signaling Pathway in Granulosa Cells from Patients with Polycystic Ovary Syndrome
}

\section{You Li}

Jiangxi Maternal and Child Health Hospital

\section{Guohui Xiong}

Jiangxi Maternal and Child Health Hospital

Jun Tan

Jiangxi Maternal and Child Health Hospital

Shudi Wang

Jiangxi Maternal and Child Health Hospital

\section{Qiongfang Wu}

Jiangxi Maternal and Child Health Hospital

Lei Wan

Jiangxi Maternal and Child Health Hospital

ZiYu Zhang ( $\nabla$ airity@163.com )

Jiangxi Maternal and Child Health Hospital

\section{Ouping Huang}

Jiangxi Maternal and Child Health Hospital

\section{Research}

Keywords: PCOS, Hedgehog, Ihh, Ptch2, Gli

Posted Date: August 6th, 2020

DOI: https://doi.org/10.21203/rs.3.rs-53354/v1

License: (1) (1) This work is licensed under a Creative Commons Attribution 4.0 International License. Read Full License 


\section{Abstract}

The molecular mechanism that triggers polycystic ovary syndrome (PCOS) is mysterious. Abnormal development of ovarian granulosa cells(GCs) is one of the causes of PCOS. Herein, we carried out RNAseq to detect the different gene expression levels in ovarian GCs between 3 patients with PCOS and 4 normal controls, and found that Hedgehog signaling pathway $(\mathrm{Hh})$ members, Ihh and Ptch2 were abnormally highly expressed in the PCOS group. To further verify the above results, GCs from 22 patients with PCOS and 21 controls with normal ovulation were collected to perform the RT-PCR analysis. The qPCR results also indicated that the expression levels of other Hh signaling pathway downstream members, Ptch1, Gli1, and Gli2 in the PCOS group were significantly higher than those in the control group. Besides, the expression of TNF-a mRNA in PCOS patients was higher than that in the control group. Finally, the Hh signaling pathway inhibitor, cyclopamine, can decrease the apoptosis of PCOS ovarian granulosa cells. These results suggest that abnormally activated Hh signaling pathway, especially lhh signal, may have a profound influence on PCOS.

\section{Introduction}

Polycystic ovary syndrome (PCOS) is one of the most common endocrine disorders in women, which affects $10 \%$ reproductive age women(1). The Rotterdam Criteria requires women to conform to two of three following symptom: oligoovulation or anovulation, clinical or biochemical hyperandrogenism and polycystic ovaries (PCO)(2). In the late development stage of PCOS, the complication associated with metabolic diseases such as diabetes, abdominal adiposity, high cholesterol and hypertension most occur(3-5). Therefore, the PCOS patients need a long term therapy to avoid the consequence. But the etiology, especial molecular mechanism and pathogenesis are still unclear.

Folliculogenesis, a highly coordinated event in the development and release of oocytes, is disrupted in PCOS. In PCOS women, excessively primordial follicles were recruited and subsequent development is arrested at the early preantral stage, resulting in the formation of multiple cysts(6). Increased GnRH pulses favor promoted LH production, which along with excess insulin stimulates ovarian theca cells to produce more androgen resulting in cessation of follicular growth and dominant follicle selection, thus affecting ovulation. Therefore, one of the most important symptoms of PCOS is the disordered follicular development. However, the precise molecular defects of follicular development in PCOS still remain unknown. The process of follicular development was accurately regulated by the communication among the oocyte, granulose cells (GCs) and theca cells(7-9), which involving several signaling pathways(10-14).

The hedgehog $(\mathrm{Hh})$ gene was first cloned in Drosophila(15). It plays a very important role in the embryonic development and remodeling processes of adult tissues(16). Hh signaling regulates cell fate determination, proliferation and differentiation(17). Moreover, over-activation of Hh signaling is associated with the tumorigenesis(18-21). In mammals, the Hh pathway consists of three Hh ligands, 
indian (Ihh), desert (Dhh), and sonic (Shh). The membrane receptors are patched (Ptch1,2) and transmembrane signal transducer protein smoothened (SMO)(22). In the absence of ligand binding, PTCH maintains SMO in an inactive state. Binding of Hh ligand to PTCH relieves inhibition of SMO, and allows the activation of $\mathrm{Hh}$-induced intracellular transcriptional effectors Glioma-associated oncogene homolog (Gli-1, 2, 3), leading to the induction of target gene expression(23).

Recently, expression of components in the Hh pathway were observed in both GCs and residual ovarian tissue, and the expression were changed in response to the stages of follicular development in human postnatal ovaries(24). Here, we present evidence that significant abnormal activation of Hedgehog signaling pathway is identified in PCOS patients. The above results indicats that Hh signaling pathway may be associated with follicular development and PCOS.

\section{Materials And Methods}

\section{Patients selection}

A prospective case-control study and qPCR analysis were designed including 22 patients with PCOS (PCOS group) and 21 patients with regular menstrual cycles (control group) from December 2016 to March 2017 in Jiangxi Maternal and Child Health Hospital. In addition, RNA-seq analysis was performed on the ovarian granular cell layer tissues from 3 PCOS patients and 4 controls. These patients received intracytoplasmic sperm injection (ICSI) and were between the ages of 20 and 34 years and weighed at least $40 \mathrm{~kg}$. PCOS patients were diagnosed based on the Rotterdam criteria, including oligoovulation or anovulation and $\mathrm{PCO}(2)$. In the control group, patients were selected including normal ovulation, absence of hirsutism and acne, absence of PCO on sonography, and normal hormonal parameters. All the patients were also excluded from the study if they had a history of unilateral oophorectomy, recurrent spontaneous abortion (defined as three of more previous spontaneous pregnancy losses), congenital or acquired uterine malformations, abnormal results on parental karyotyping, endometriosis, hyperprolactinemia and thyroid dysfunction. All selected patients followed the voluntary principle and signed informed consent. This experiment conforms to the Helsinki principle and is approved by our ethics committee

\section{Blood sampling and sex hormone measurement}

Blood samples were collected on the third day of the menstrual cycle and on the day of HCG injection, subsequently centrifuged at 4000rpm for $1 \mathrm{~min}$. The serum was used for the quantitative determination of sex hormone (follicle-stimulating hormone $(\mathrm{FSH})$, luteinizing hormone $(\mathrm{LH})$, estradiol $\left(\mathrm{E}_{2}\right)$, prolactin (PRL) and testosterone (TES) level by chemiluminescent enzyme immunoassay using Automated Enzyme Immunoassay Analyzer (AIA-2000ST, TOSOH CORPORATION). The above experiment was repeated three times, and the results come from the clinical test results. 


\section{Controlled ovarian stimulation}

Follicular aspirates were collected during oocyte retrieval following published procedures and ovarian stimulation was the use of a prolonged protocol $(25,26)$. Briefly, gonadotropin-releasing hormone agonist (GnRH-a, Ipsen, Boulogne-Billancourt, France) was used in the second or three day of menstrual cycle for pituitary down-regulation. Gonadotropin stimulation were started after 28 or 38 days following the criteria: no ovarian cysts $>8 \mathrm{~mm}, \mathrm{E}_{2}<50 \mathrm{pg} / \mathrm{ml}, \mathrm{FSH}<5 \mathrm{IU} / \mathrm{L}, \mathrm{LH}<5 \mathrm{IU} / \mathrm{L}$. Initial, patients received 75$112.5 \mathrm{IU} / \mathrm{d}$ of recombinant human FSH (Merck-Serono, Darmstadt, Germany) according to the patient's age, body mass index (BMI), serum basal FSH levels, LH levels, $E_{2}$ levels and antral follicle count. The time and dose of recombinant human FSH were adjusted according to ovarian response as monitored by serum $E_{2}$ levels and vaginal ultrasound. When the dominant follicle was $\geq 19 \mathrm{~mm}$ in diameter or at least 3 follicles were $\geq 17.5 \mathrm{~mm}$ in diameter, recombinant human FSH was stopped, and a single injection of 6000-8000 IU of hCG (Merck-Serono, Darm-stadt, Germany) was administered. Oocytere-trieva I was perfor med 36-40 hours later under transvaginal ultrasound guidance.

\section{Human GCs collection and cell culture}

Follicular fluids were centrifuged at $2000 \mathrm{rpm}$ for $5 \mathrm{~min}$. The cells were resuspended with DMEM/F 12 (Life Technologies, Carlsbad, CA, USA) medium and transferred to a 50\% (volume fraction) Percoll gradient (Sigma-Aldrich, St. Louis, MO, USA); they were centrifuged at $4000 \mathrm{rpm}$ for 20 min to purify human GCs from any red blood cells. After washing and recentrifugation, sheets of human GCs were digested with trypsinat a 1:1 ratio for 4 min to separate them. The GCs were removed using a pipette and washed with phosphate buffered saline (PBS). 10\% FBS DMEM / F12 culture medium at a 2:1 ratio were added to terminate the digestion, centrifuged at $1500 \mathrm{rmp}$ for 3 minutes. GCs were resuspended with $1 \times$ PBS at a 1:5 ratio, centrifuged at 1500 rmp for 3 minutes. After discarding the supernatant, added $1 \mathrm{ml}$ of $10 \%$ FBS DMEM / F12 culture medium, resuspended, inoculated in a dish, and placed in a $37^{\circ} \mathrm{C}, 5 \%$ $\mathrm{CO} 2$ incubator. After observing the morphology of the granulocytes under the microscope, changed the fluid per 24 hours. The cells at indicated time were stored at $-80^{\circ} \mathrm{C}$ for future analysis.

\section{FSHR immunohistochemical staining in human GCs}

After the GCs are cultured for 3 days, the fixed cell slides are washed with PBS for 3 times, and fixed with $4 \%$ paraformaldehyde for 30 min, washed with PBS for 3 times. Incubated in $3 \% \mathrm{H}_{2} \mathrm{O}_{2}$ deionized water for 10 minutes to block the effect of endogenous peroxidase, washed in 1xPBS for 2 times. Drop anti-rabbit anti-human FSHR polyclonal antibody (1:100) dropwise at $4{ }^{\circ} \mathrm{C}$ overnight, washed in PBS for 2 times. Added reagent 1 (polymer helper) dropwise, incubated at room temperature for $20 \mathrm{~min}$, and washed in 1XPBS for 2 times. Added reagent 2 (polyperoxidase-anti-mouse / rabbit lgG) dropwise and incubated at room temperature. Finally, DAB was added dropwise for color reaction (control reaction time under microscope). Rinsed thoroughly with tap water, counterstained with hematoxylin for $1 \mathrm{~min}$, dehydrated 
with conventional gradient alcohol, transparent xylene and seal with neutral resin. As a negative control, 1xPBS was used instead of the primary antibody. The cells with yellow staining on the cell membrane were FSHR positive cells, and the number of positive cells in 10 high-power $(\times 400)$ visual fields was randomly counted.

\section{$R N A-s e q$ and $q P C R$}

For RNA-seq, total RNA were extracted from 4 normal tissues and 3 PCOS tissues by using the RNAiso reagent (TaKaRa, Shiga, Japan). The Library was validated on the Agilent Technologies 2100 bioanalyzersubjected to deep sequencing onlllumina HiSeq 2000 (50-bp single-read sequencing), and analyzed at BGI Genomics Co., Ltd. For RT-qPCR analysis, the GCs total RNA from 22 patients with PCOS and 21 controls were carried out by using the PrimeScript RT reagent Kit (TaKaRa). Standard qPCR was carried out with the following primers: hsa-RT-Ptch1 (5'-GCTGCACTACTTCAGAGACTGG-3' and 5'CACCAGGAGTTTGTAGGCAAGG-3'), hsa-RT-Gli1 (5'-AGCCTTCAGCAATGCCAGTGAC-3' and 5'GTCAGGACCATGCACTGTCTTG-3'), hsa-RT-Gli2 (5'-GTCAGAGCCATCAAGACCGAGA-3' and 5'GCATCTCCACGCCACTGTCATT-3'), hsa-RT-Gli3 (5'-TCAGCAAGTGGCTCCTATGGTC-3' and 5'GCTCTGTTGTCGGCTTAGGATC-3') and has-RT-actin (5'-ACCTTCTACAATGAGCTGCG-3' and 5'CCTGGATAGCAACGTACATGG-3'). Real-time PCR was carried out using the FastStart SYBR Green Master mix (Roche) on a 7500 Real-Time PCR System (Applied Biosystems, Grand Island, NY). The actin was used as an internal control. The results were presented as fold change, calculated using the $2^{-\triangle C T}$ method, and a ratio of expression in the PCOS relative to the Controls less than 1.0 was considered as low.

\section{Statistical analysis}

Statistical analysis was performed by using the SPSS 17.0 software package. Data were shown as mean \pm SEM. The t-test was used for comparison between measurement data groups and the $\chi 2$ test was used for count data groups. Each experiment was repeated at least 3 times. ${ }^{*} p<0.05$ was considered as statistically significant.

\section{Results}

\section{Ihh and Ptch2 were upregulated in PCOS}

In order to explore the molecular mechanism of PCOS, we used RNA-seq analysis to detect the difference in gene expression levels between the PCOS (PT) group and the normal control group (NT). The RNA-seq result showed that there were total 673 differentially expression genes(DEG). Among them, 296 genes were up-regulated and 377 genes were down-regulated (Fig.1). Moreover, pathway functional enrichment results of NT-VS-PT.DEGseqPathway indicated that immune system related genes occupied an important part of DEG (Fig.2). Interestingly, among DEGs, we found that the Hh pathway member, ligand Ihh and the 
receptor Ptch2 (also the target genes downstream of the Hh pathway) are highly expressed in the PCOS group (Table.1). Next, we would use more clinical samples for subsequent analysis.

Table 1

Expression of a part of DEGs hierarchical clustering analysis results of PCOS patients and control group

\begin{tabular}{|llllllll|}
\hline Symbol & GenelD & Length & NT & PT & log2(PT/NT) & Up/Down & p-value \\
TNXA & 7146 & 2783 & 168.4 & 354.2 & 1.4 & Up & $<0.001$ \\
IL4I1 & 259307 & 1798 & 168.2 & 350.4 & 1.4 & Up & $<0.001$ \\
S100B & 6285 & 1135 & 357 & 572 & 1.0 & Up & $<0.001$ \\
\hline IHH & 3549 & 2074 & 20 & 129 & 3.0 & Up & $<0.001$ \\
APOD & 347 & 1148 & 878 & 1984 & 1.5 & Up & $<0.001$ \\
SDS & 10993 & 1620 & 410 & 1322 & 2.0 & Up & $<0.001$ \\
\hline PTCH2 & 8643 & 3840 & 1803.9 & 3109.2 & 1.1 & Up & $<0.001$ \\
\hline CTSH & 1512 & 1532 & 1616 & 2873 & 1.2 & Up & $<0.001$ \\
\hline LILRB5 & 10990 & 2395 & 361.4 & 1231.8 & 2.1 & Up & $<0.001$ \\
\hline CCL4 & 6351 & 667 & 2042.2 & 3323.8 & 1.1 & Up & $<0.001$ \\
\hline C4B & 721 & 5444 & 838.9 & 1840.7 & 1.5 & Up & $<0.001$ \\
\hline
\end{tabular}

\section{General conditions}

In the general comparison of the two groups of patients(22 PCOS and 21 Controls), the age, infertility, $\mathrm{BMI}$, blood base FSH, E2, PRL of the PCOS group were not statistically significant compared with the control group. Comparing the blood LH between the two groups, the PCOS group (7.30 $\pm 3.59 \mathrm{IU} / \mathrm{L})$ was significantly higher than the control group $(4.43 \pm 2.35 \mathrm{IU} / \mathrm{L})$, which was statistically significant. The blood TES in the PCOS group $(38.28 \pm 20.30 \mathrm{nmol})$. / L) was significantly higher than that in the control group (26.34 $\pm 10.79 \mathrm{nmol} / \mathrm{L}$ ), the two groups were statistically significant, $\mathrm{p}<0.05$ (Table.2). 
Table 2

Comparison of general conditions between PCOS group and control group

\begin{tabular}{|llll|}
\hline Characteristics & PCOS $(\mathrm{n}=22)$ & Control $(\mathrm{n}=21)$ & $P$ \\
\hline Age (years) & $27.64 \pm 2.82$ & $28.95 \pm 2.77$ & $>0.05$ \\
\hline Infertility duration (years) & $4.05 \pm 2.44$ & $3.14 \pm 2.29$ & $>0.05$ \\
\hline BMI $\left(\mathrm{Kg} / \mathrm{m}^{2}\right)$ & $22.03 \pm 3.25$ & $22.67 \pm 3.47$ & $>0.05$ \\
\hline Basal FSH (IU/L) & $5.42 \pm 1.09$ & $5.93 \pm 1.36$ & $>0.05$ \\
\hline Basal LH (IU/L) & $7.30 \pm 3.59$ & $4.43 \pm 2.35$ & $<0.05$ \\
\hline Basal E $2(\mathrm{pg} / \mathrm{mL} \otimes$ & $39.05 \pm 18.67$ & $40.11 \pm 17.00$ & $>0.05$ \\
\hline PRL $(\mathrm{ug} / \mathrm{L})$ & $19.02 \pm 13.24$ & $17.65 \pm 9.84$ & $>0.05$ \\
\hline $\mathrm{T}(\mathrm{nmol} / \mathrm{L})$ & $38.28 \pm 20.30$ & $26.34 \pm 10.79$ & $<0.05$ \\
\hline
\end{tabular}

Note: The date were expressed as mean + SD.

\section{Clinical outcomes}

In the general comparison of IVF-ET treatment between the two groups of patients, the endometrial thickness of HCG on the PCOS group $(10.6 \pm 1.7 \mathrm{~mm})$ was significantly lower than that of the control group $(11.9 \pm 2.0 \mathrm{~mm})$. Other indicators such as total $\mathrm{Gn}$ amount, total $\mathrm{Gn}$ days, HH day LH, E2 and P were not statistically significant in the two groups. The MII egg rate of the PCOS group $(60.7 \pm 28.9 \%)$ was significantly lower than that of the control group $(80.4 \pm 13.6 \%)$, and the two groups were statistically significant. The 2PN fertilization rate of the PCOS group $(61.7 \pm 20.8 \%)$ was significantly lower than the control group $(76.9 \pm 18.6 \%)$, the two groups were statistically significant. Other indicators such as the number of eggs obtained, the number of embryos available and the rate of superior embryos were not statistically significant between the two groups(Table.3). 
Table 3

Comparison of clinical outcomes between PCOS group and control group

\begin{tabular}{|llll|}
\hline Variables & PCOS & control & $P$ \\
\hline Dosage of Gn (IU) & $1916 \pm 944$ & $2144 \pm 925$ & $>0.05$ \\
\hline Duration of Gn (days) & $12.7 \pm 2.6$ & $11.6 \pm 1.7$ & $>0.05$ \\
\hline $\begin{array}{l}\text { Endometrial thickness } \\
\text { on HCG day (mm) }\end{array}$ & $10.6 \pm 1.7$ & $11.9 \pm 2.0$ & $<0.05$ \\
\hline LH on HCG day (IU/L) & $1.1 \pm 0.6$ & $0.9 \pm 0.5$ & $>0.05$ \\
\hline E2 on HCG day (pmol/I) & $2269 \pm 1155$ & $2510 \pm 1110$ & $>0.05$ \\
\hline P on HCG day (nmol/I) & $1.1 \pm 0.4$ & $1.0 \pm 0.5$ & $>0.05$ \\
\hline No of oocytes retrieved & $16 \pm 9$ & $14 \pm 6$ & $>0.05$ \\
\hline MII oocyte rate (\%) & $60.7 \pm 28.9$ & $80.4 \pm 13.6$ & $<0.05$ \\
\hline Fertilization rate (\%) & $61.7 \pm 20.8$ & $76.9 \pm 18.6$ & $<0.05$ \\
\hline Cleavage rate (\%) & $97.7 \pm 5.9$ & $92.7 \pm 12.0$. & $>0.05$ \\
\hline No of Embryo & $3.4 \pm 1.6$ & $4.1 \pm 2.4$ & $>0.05$ \\
\hline Good quality embryo rate (\%) & $2.1 \pm 1.8$ & $3.1 \pm 2.8$ & $>0.05$ \\
\hline
\end{tabular}

Note: The date were expressed as mean + SD.

\section{Expression of Hh family members in GCs from PCOS group and control Group}

As we know that PCOS are often accompanied with abnormal follicular development, therefore, we consider that whether the disordered $\mathrm{Hh}$ signaling pathway is contributed to the PCOS-related abnormal follicles. Then we isolated and purified granulosa cells from 22 PCOS and 21 Control samples. We performed FSH staining after 3-5 days of cell culture (Fig.3). After identification, total RNA was extracted for qPCR detection. Next, we compared the mRNA levels of Gli1, Gli2, Gli3 and Ptch1 in PCOS and nonPCOS groups undergoing IVF treatment to explore the potential role of Hh signaling pathway in PCOSrelated abnormal follicles. We tested the expression of Hh family members of GCs in both PCOS and controls. The levels of Gli1 mRNA, Ptch1 mRNA and Gli2 mRNA were significantly higher in PCOS group than those in the control group, while the expression of Gli3 mRNA had no significant difference between the two groups (Fig.4). These results indicate that the abnormality of the Hh pathway has a potential role in promoting the development of PCOS. 


\section{The expression of TNF-a mRNA in PCOS patients was higher than that in the control group}

Because TNF- $a$ can induce apoptosis, and the RNA-seq results also showed that the expression of TNF-a in the PCOS group was higher than that in the control group (Table.4), and it was statistically significant. Therefore, we used the second part of the mRNA of the granulosa cells of the two groups of patients to perform qPCR experiments to verify the above RNA-seq data. The results showed that in the PCOS group, the mRNA level of TNF-a was significantly higher than that of the control group, and it was statistically significant (Fig.5). The above results indicate that the expression of TNF-a mRNA in PCOS patients is higher than that in the control group.

Table 4

Expression of a part of DEGs hierarchical clustering analysis results of PCOS patients and control group

\begin{tabular}{|lllllll|}
\hline Symbol & GenelD & Length & NT & PT & log2(PT/NT) & Up/Down \\
\hline 54209 & TREM2 & 266 & 810 & 1.96 & Up & $<0.001$ \\
\hline 81035 & COLEC12 & 497 & 1119 & 1.52 & Up & $<0.001$ \\
\hline 7124 & TNF & $\mathbf{8 4 8}$ & 1547 & $\mathbf{1 . 2 2}$ & Up & $<0.001$ \\
\hline 23237 & ARC & 407 & 973 & 1.61 & Up & $<0.001$ \\
\hline
\end{tabular}

\section{Inhibition of Hh signaling pathway can decrease the apoptosis of PCOS ovarian granulosa cells}

Because TNF-a can induce apoptosis, and TNF expression and Hh signal increase in GCs of PCOS patients (Fig.4 and 5), suggesting that the activation of Hh pathway may be related to the apoptosis of GCs. In order to detect whether excessive activation of the Hh signaling pathway can promote cell apoptosis, we used PCOS ovarian granulosa cells for apoptosis detection experiments. The cells were treated with CPA, an inhibitor of the Hh signaling pathway, for 24 hours, and then the cell apoptosis was detected by flow cytometry using Annexin V-FITC and prodiduim iodine (PI) double staining. The results showed that PCOS ovarian granulosa cells were added to CPA After that, cell apoptosis was significantly reduced (Fig.6). The above results indicate that inhibition of $\mathrm{Hh}$ signaling pathway can simultaneously reduce the apoptosis of PCOS ovarian granulosa cell.

\section{Discussion}

In our research, PCOS patients were diagnosed based on the Rotterdam criteria including oligoovulation or anovulation and PCO. This study was a matching study and no statistically significant difference was 
found in age, infertile period, or BMI in comparing the two groups as we showed in the Table 1. The PCOS group had higher level of Basal LH and T, which were consisted with their abnormal endocrine results (36). Previous studies have found that PCOS patients were often accompanied with aberrant follicles and unsatisfactory fertilization rate(25-27). We also analyzed clinical outcomes of PCOS groups and control. As shown in Table 2, the MII oocyte rate and fertilization rate were remarkably reduced in PCOS group when compared with the control group $(60.7 \pm 28.9$ vs. $80.4 \pm 13.6, \mathrm{P}<0.05 ; 61.7 \pm 20.8$ vs. $76.9 \pm 18.6, \mathrm{P}$ $<0.05)$. In line with previous studies, our data also showed a high rate of immature oocyte and low rate of fertilization in PCOS patients.

Patients with PCOS infertility can usually obtain normal pregnancy through clinical routine treatment methods such as drug promotion, surgery, lifestyle adjustment and weight loss. However, for patients with refractory PCOS or patients with other infertility factors and male factors, they will choose assisted reproductive technology (ART). In the treatment of PCF patients with IVF-ET, the high levels of LH and androgens in the body tend to cause follicular atresia and premature lutealization. The quality of eggs and embryos formed is relatively poor, the pregnancy rate is reduced, and the abortion rate is relatively high. It is also possible to restrict the transfer of embryos by causing the endometrium to express the progesterone receptor prematurely and convert it into the secretory endometrium, thereby reducing the implantation rate and clinical pregnancy rate (25-27) \{Tan, 2016 \#43\}. In addition, a large number of small follicles remain in the ovaries of patients with PCOS. For a long period of time under high androgen levels, a high level of gonadotropin is required to start. Once activated, a large number of follicles develop at the same time, and it is easy to form excessive reactions. Lead to the occurrence of ovarian hyperstimulation syndrome (OHSS)(28). The common ovulation-promoting programs of PCOS mainly include long programs, ultra-long programs, antagonist programs, and microstimulation programs (29). In our study, patients in both groups were treated with $\mathrm{COH}$ after down-regulation with an ultralong protocol. Studies have shown that prolonged down-regulation can significantly improve the thickness, morphology, and blood flow of the endometrium, thereby increasing the implantation rate and pregnancy rate (27). At the same time, it can reduce the concentration of pelvic inflammatory cytokines and improve pelvic cavity. The environment is favorable for embryo implantation (30).

In our study comparing IVF-ET treatment in general, the endometrial thickness of HCG in the PCOS group $(10.6 \pm 1.7 \mathrm{~mm})$ was significantly lower than that in the control group $(11.9 \pm 2.0 \mathrm{~mm})$, and the results were statistically significant. Other indicators such as total Gn amount, total Gn days, HH day LH, E2 and $P$ were not statistically significant in the two groups. Studies have shownthat an appropriate thickness of the endometrium is important for embryo implantation. When the endometrial thickness is> $14 \mathrm{~mm}$ on the day of hCG injection, the IVF implantation rate and pregnancy rate are reduced, and the endometrial thickness is $<6$. At $\sim 7 \mathrm{~mm}$, the embryo implantation rate and pregnancy rate are affected, and the miscarriage rate increases. In this study, the average endometrial thickness of PCOS patients on HCG day was $10.6 \mathrm{~mm}$ lower than the normal control group's $11.9 \mathrm{~mm}$, but all were within the normal range and had no obvious clinical significance. 
In IVF-ET treatment, the MII egg rate is the ratio of mature eggs. After the sperm and the egg meet, the sperm enters the egg, and the nucleus of the egg and the nucleus of the sperm will form a pronucleus, respectively. The two pronucleus are called "2PN", indicating that the egg is fertilized normally. The 2PN fertilization rate represents the rate of normal fertilization of the egg. In our study, the MIl egg rate of the PCOS group $(60.7 \pm 28.9 \%)$ was significantly lower than that of the control group (80.4 $\pm 13.6 \%)$, which was statistically significant; the 2 PN fertilization rate $(61.7 \pm 20.8 \%)$ of the PCOS group was significant Compared with the control group $(76.9 \pm 18.6 \%)$, the two groups were statistically significant, consistent with the results of previous studies of PCOS. At the same time, other indicators such as the number of eggs obtained, the number of available embryos, and the rate of superior embryos were also consistent with other studies. There was no statistical significance in the two groups.

The Hh signaling pathway is proved to play a crucial role in embryonic development of mammalian including human. GCs from growing follicles in mouse ovary acting as a source of Hh signaling was first reported in 2005(22), which demonstrated that Hh family were expressed on the GCs from primary to antral stages of follicular development in postnatal ovaries of human, respectively $(24,31)$. These results implied that $\mathrm{Hh}$ signaling played an important role in the communication between the cellular compartments that perform gametogenesis, steroidogenesis and ovarian vasculature(22, 24, 32). Activation of $\mathrm{Hh}$ signaling regulated follicle growth and GCs proliferation at least one of the potential targets(24). These studies suggested a sequential requirement for Hh signaling pathway in ovarian follicular development. Previous studies also found that patients with PCOS were often accompanied with follicular dysplasia $(6,33)$. Therefore, we speculate that maybe there is a relationship between $\mathrm{Hh}$ components expression and PCOS.

The three Hh ligands (Ihh, Shh and Dhh), the two receptors (Ptch1 and Ptch2) and the mediator of HH signaling (Smo), are expressed in GCs and in corpora lutea from pseudopregnant mice(24). In addition, the transcription factor, Gli1, Gli2 and Gli3 are expressed in all ovarian tissues(34-36). The ovarian $\mathrm{Hh}$ signaling system could be involved in the proliferation of GCs under certain conditions(31). Expressions of a number of Hh genes in GCs that are known to be important for ovulation were no difference between mutants and controls(35). Some studies suggest that an association exists between modulation of the Hh pathway and selection of the dominant follicle(s)(36). In order to discuss the relationship between $\mathrm{Hh}$ signaling activity and PCOS which were accompanied with follicular dysplasia, we measured the components of the Hh pathway in GCs. We found that PCOS groups showed higher mRNA levels of Ptch1, Gli1 and Gli2, when compared to control groups; while the level of Gli3 mRNA had no significant difference. Ptch 1 is a key component of the Hh signaling pathway, which controls cell fate determination during development(37). Ptch1 mutations cause derepression of target genes, cell fate changes, and excessive growth in some tissues(38). Gli1 lacks the N-terminal repressor domain and functions exclusively as an activator. The Gli1 gene is also a target of Hh signaling and thus acts to amplify the response to the signal $(35,39)$. Thus, the higher mRNA levels of Ptch1 and Gli1 confirmed that Hh signaling pathway is aberrant activated in the GCs of PCOS patients than control. In addition, Gli2 and Gli3 proteins contain both activator and repressor domains and undergo proteasome-dependent proteolytic cleavage(16). In our research, although Gli3 acting mainly as a repressor was no difference in 
GCs from these two groups, Gli2 appearing to function mostly as an activator was significantly higher in GCs of PCOS.

Admittedly, the communication between oocytes and GCs is important for the development of follicular, in which GCs secrete various kinds of nutritional factors to promote oocyte growth, simultaneously, oocytes product several factors to regulate GCs development $(7,8,33)$. Therefore, the function status of GCs is often considered as the mirror of oocyte quality. It is well known that PCOS patients are often accompanied with aberrant GCs in follicles(7-9). The Hh signaling pathway was higher activated in GCs of PCOS than control, which implied the aberrant activation of Hh signaling pathway was related to abnormal follicular development in PCOS patients.

\section{Conclusions}

Herein, we first demonstrate that aberrant activation of Hh signaling pathway in GCs is related to abnormal follicular development in PCOS patients. These findings provide a basis for future investigations to define cell-specific response to $\mathrm{Hh}$ signaling in the follicle and to determine how the pathway modulates follicle development.

\section{Abbreviations}

PCOS: polycystic ovary syndrome

GCs: granulosa cells

Hh: Hedgehog

SMO: smoothened

FSH: follicle-stimulating hormone

LH: luteinizing hormone

$\mathrm{E}_{2}$ :estradiol

PRL: prolactin

TES: testosterone

GnRH-a: gonadotropin-releasing hormone agonist

\section{Declarations}

\section{Ethics approval and consent to participate}


The patient signed a written informed consent form prior to recruitment. This study is in line with the Helsinki Declaration and approved by the Ethics Review Body Committee of the Jiangxi Maternal and Child Health Hospital.

\section{Consent for publication}

Not applicable.

\section{Availability of data and materials}

The datasets used and/or analysed during the current study are available from the corresponding author on reasonable request.

\section{Competing interests}

The authors declare that they have no competing interests.

\section{Funding}

This work was supported by the National Natural Science Foundation of China (No. 81771559) to O.P.H, (No. 81660736) to L.W and Class C Key Research and Development Program of Jiangxi Province (No. 20181BBG70009) to Z.Y.Z.

\section{Authors' contributions}

O.P.H and Y.L were responsible for the experimental design and drafting the manuscript. Y.L and S.D.W were involved in carrying out the experiments. G.H.X and Z.Y.Z were responsible for analyzing and interpreting the data with the assistant of Q.F.Wand L.W. J.T performed the statistical analysis and edited the manuscript.

\section{Acknowledgements}

The authors wish to thank members of the Huang laboratory for technical assistance.

\section{References}

1. Norman RJ, Dewailly D, Legro RS, Hickey TE. Polycystic ovary syndrome. Lancet 2007;370:685-697. 
2. Rotterdam EA-SPCWG. Revised 2003 consensus on diagnostic criteria and long-term health risks related to polycystic ovary syndrome. Fertil Steril 2004;81:19-25.

3. Setji TL, Brown AJ. Polycystic ovary syndrome: update on diagnosis and treatment. Am J Med 2014;127:912-919.

4. Tandulwadkar SR, Lodha PA, Mangeshikar NT. Obstetric complications in women with IVF conceived pregnancies and polycystic ovarian syndrome. J Hum Reprod Sci 2014;7:13-18.

5. Japur CC, Diez-Garcia RW, de Oliveira Penaforte FR, de Sa MF. Imbalance Between Postprandial Ghrelin and Insulin Responses to an Ad Libitum Meal in Obese Women With Polycystic Ovary Syndrome. Reprod Sci 2014;21:1020-1026.

6. Diamanti-Kandarakis E. Polycystic ovarian syndrome: pathophysiology, molecular aspects and clinical implications. Expert Rev Mol Med 2008;10:e3.

7. Nilsson E, Skinner MK. Cellular interactions that control primordial follicle development and folliculogenesis. J Soc Gynecol Investig 2001;8:S17-20.

8. Parrott JA, Skinner MK. Thecal cell-granulosa cell interactions involve a positive feedback loop among keratinocyte growth factor, hepatocyte growth factor, and Kit ligand during ovarian follicular development. Endocrinology 1998;139:2240-2245.

9. Zhang CL, Wang H, Yan CY, Gao XF, Ling XJ. Deregulation of RUNX2 by miR-320a deficiency impairs steroidogenesis in cumulus granulosa cells from polycystic ovary syndrome (PCOS) patients. Biochem Biophys Res Commun 2017;482:1469-1476.

10. Xing Y, Liu YX, Liu X, Wang SL, Li P, Lin XH, Sui CL, et al. Effects of Gui Zhu Yi Kun formula on the P53/AMPK pathway of autophagy in granulosa cells of rats with polycystic ovary syndrome. Exp Ther Med 2017;13:3567-3573.

11. Wu XQ, Wang YQ, Xu SM, Liu JF, Bi XY, Wang ZQ, Zhang JP. The WNT/beta-catenin signaling pathway may be involved in granulosa cell apoptosis from patients with PCOS in North China. $J$ Gynecol Obstet Hum Reprod 2017;46:93-99.

12. Nasri K, Hantoushzadeh S, Aghadavod E, Taghizadeh M, Asemi Z. The Effects of Omega-3 Fatty Acids Supplementation on Gene Expression Involved in the Insulin and Lipid Signaling Pathway in Patients with Polycystic Ovary Syndrome. Horm Metab Res 2017;49:446-451.

13. Jiang SW, Xu S, Chen H, Liu X, Tang Z, Cui Y, Liu J. Pathologic significance of SET/I2PP2A-mediated PP2A and non-PP2A pathways in polycystic ovary syndrome (PCOS). Clin Chim Acta 2017;464:155159.

14. Li T, Mo H, Chen W, Li L, Xiao Y, Zhang J, Li X, et al. Role of the PI3K-Akt Signaling Pathway in the Pathogenesis of Polycystic Ovary Syndrome. Reprod Sci 2017;24:646-655.

15. Nusslein-Volhard C, Wieschaus E. Mutations affecting segment number and polarity in Drosophila. Nature 1980;287:795-801.

16. Lee RT, Zhao Z, Ingham PW. Hedgehog signalling. Development 2016;143:367-372. 
17. King PJ, Guasti L, Laufer E. Hedgehog signalling in endocrine development and disease. J Endocrinol 2008;198:439-450.

18. Rennert C, Eplinius F, Hofmann U, Johanning J, Rolfs F, Schmidt-Heck W, Guthke R, et al. Conditional loss of hepatocellular Hedgehog signaling in female mice leads to the persistence of hepatic steroidogenesis, androgenization and infertility. Arch Toxicol 2017;91:3677-3687.

19. Lubik AA, Nouri M, Truong S, Ghaffari M, Adomat HH, Corey E, Cox ME, et al. Paracrine sonic hedgehog signaling contributes significantly to acquired steroidogenesis in the prostate tumor microenvironment. Int J Cancer 2017; 140:358-369.

20. Hirotsu M, Setoguchi T, Sasaki H, Matsunoshita Y, Gao H, Nagao H, Kunigou O, et al. Smoothened as a new therapeutic target for human osteosarcoma. Mol Cancer 2010;9:5.

21. Galimberti F, Busch AM, Chinyengetere F, Ma T, Sekula D, Memoli VA, Dragnev KH, et al. Response to inhibition of smoothened in diverse epithelial cancer cells that lack smoothened or patched 1 mutations. Int J Oncol 2012;41:1751-1761.

22. Wijgerde M, Ooms M, Hoogerbrugge JW, Grootegoed JA. Hedgehog signaling in mouse ovary: Indian hedgehog and desert hedgehog from granulosa cells induce target gene expression in developing theca cells. Endocrinology 2005;146:3558-3566.

23. Hooper JE, Scott MP. Communicating with Hedgehogs. Nat Rev Mol Cell Biol 2005;6:306-317.

24. Russell MC, Cowan RG, Harman RM, Walker AL, Quirk SM. The hedgehog signaling pathway in the mouse ovary. Biol Reprod 2007;77:226-236.

25. Tan J, Wen XY, Su Q, Huang ZH, He JX, Xin CL, Wu QF, et al. Reduced expression of SCF in serum and follicle from patients with polycystic ovary syndrome. Eur Rev Med Pharmacol Sci 2016;20:50495057.

26. Ren J, Sha A, Han D, Li P, Geng J, Ma C. Does prolonged pituitary down-regulation with gonadotropinreleasing hormone agonist improve the live-birth rate in in vitro fertilization treatment? Fertil Steril 2014;102:75-81.

27. Gong F, Li X, Zhang S, Ma H, Cai S, Li J, Lin GE, et al. A modified ultra-long pituitary downregulation protocol improved endometrial receptivity and clinical outcome for infertile patients with polycystic ovarian syndrome. Exp Ther Med 2015;10:1865-1870.

28. Chen ZJ, Shi Y, Sun Y, Zhang B, Liang X, Cao Y, Yang J, et al. Fresh versus Frozen Embryos for Infertility in the Polycystic Ovary Syndrome. N Engl J Med 2016;375:523-533.

29. Xiao J, Chen S, Zhang C, Chang S. Effectiveness of GnRH antagonist in the treatment of patients with polycystic ovary syndrome undergoing IVF: a systematic review and meta analysis. Gynecol Endocrinol 2013;29:187-191.

30. Meresman GF, Bilotas MA, Lombardi E, Tesone M, Sueldo C, Baranao RI. Effect of GnRH analogues on apoptosis and release of interleukin-1 beta and vascular endothelial growth factor in endometrial cell cultures from patients with endometriosis. Hum Reprod 2003;18:1767-1771.

31. Spicer LJ, Sudo S, Aad PY, Wang LS, Chun SY, Ben-Shlomo I, Klein C, et al. The hedgehog-patched signaling pathway and function in the mammalian ovary: a novel role for hedgehog proteins in 
stimulating proliferation and steroidogenesis of theca cells. Reproduction 2009;138:329-339.

32. Ren Y, Cowan RG, Migone FF, Quirk SM. Overactivation of hedgehog signaling alters development of the ovarian vasculature in mice. Biol Reprod 2012;86:174.

33. Franks S, McCarthy MI, Hardy K. Development of polycystic ovary syndrome: involvement of genetic and environmental factors. Int J Androl 2006;29:278-285; discussion 286-290.

34. Migone FF, Ren Y, Cowan RG, Harman RM, Nikitin AY, Quirk SM. Dominant activation of the hedgehog signaling pathway alters development of the female reproductive tract. Genesis 2012;50:28-40.

35. Ren Y, Cowan RG, Harman RM, Quirk SM. Dominant activation of the hedgehog signaling pathway in the ovary alters theca development and prevents ovulation. Mol Endocrinol 2009;23:711-723.

36. Aad PY, Echternkamp SE, Sypherd DD, Schreiber NB, Spicer LJ. The hedgehog system in ovarian follicles of cattle selected for twin ovulations and births: evidence of a link between the IGF and hedgehog systems. Biol Reprod 2012;87:79.

37. Hammerschmidt M, Brook A, McMahon AP. The world according to hedgehog. Trends Genet 1997;13:14-21.

38. Ingham PW, Taylor AM, Nakano Y. Role of the Drosophila patched gene in positional signalling. Nature 1991;353:184-187.

39. Ingham PW, Nakano Y, Seger C. Mechanisms and functions of Hedgehog signalling across the metazoa. Nat Rev Genet 2011;12:393-406.

\section{Figures}

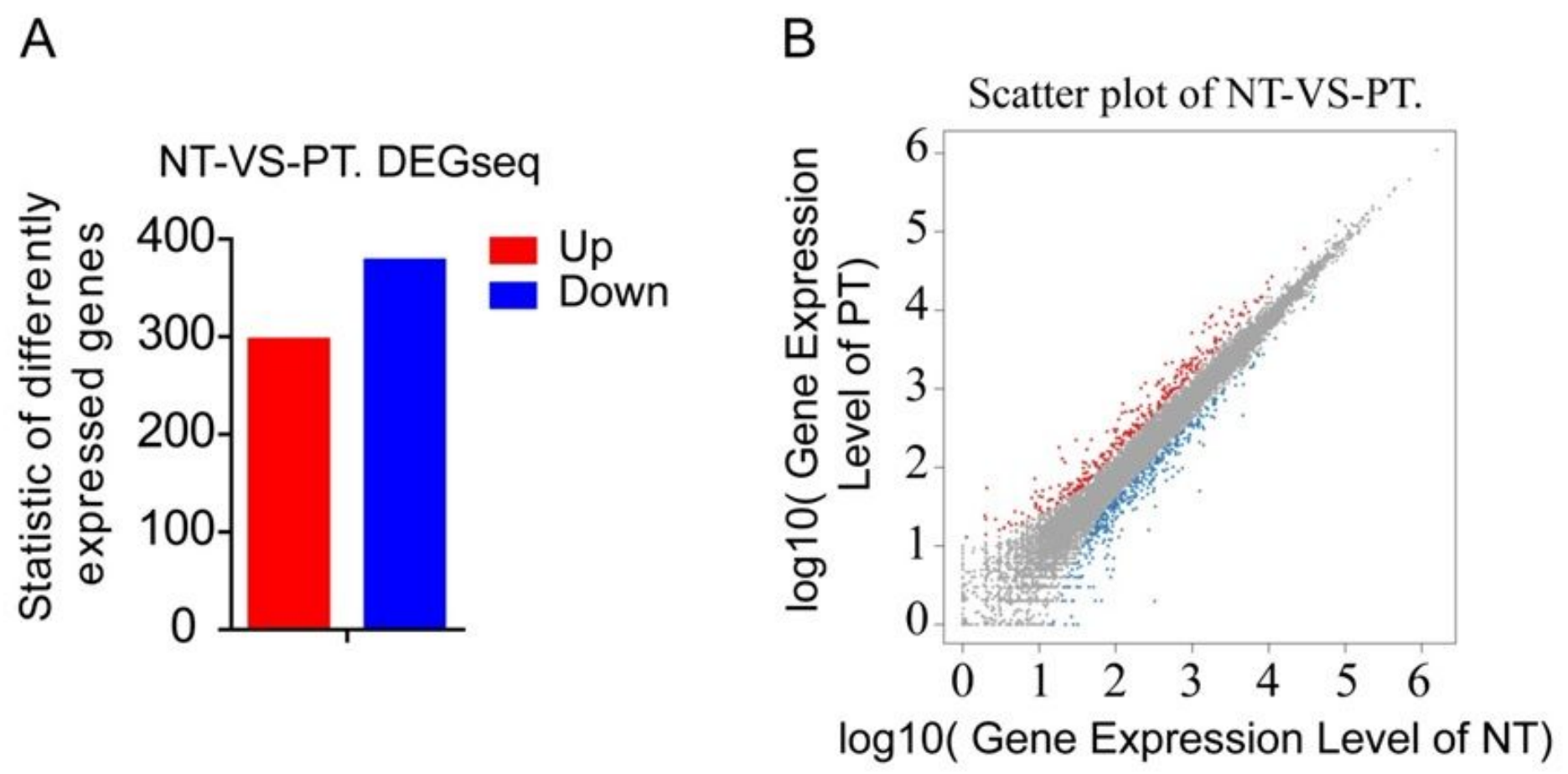


Figure 1

Summary of DEGs by RNA-seq. (A) X axis represents comparison method between each group. $Y$ axis represents DEG numbers. Red color represents up-regulated DEGs. Blue color represents down-regulated DEGs. (B) Scatter plot of DEGs. XY axis represents log10 transformed gene expression level, red color represents the up-regulated genes, blue color represents the down-regulated genes, gray color represents the non-DEGs.

A

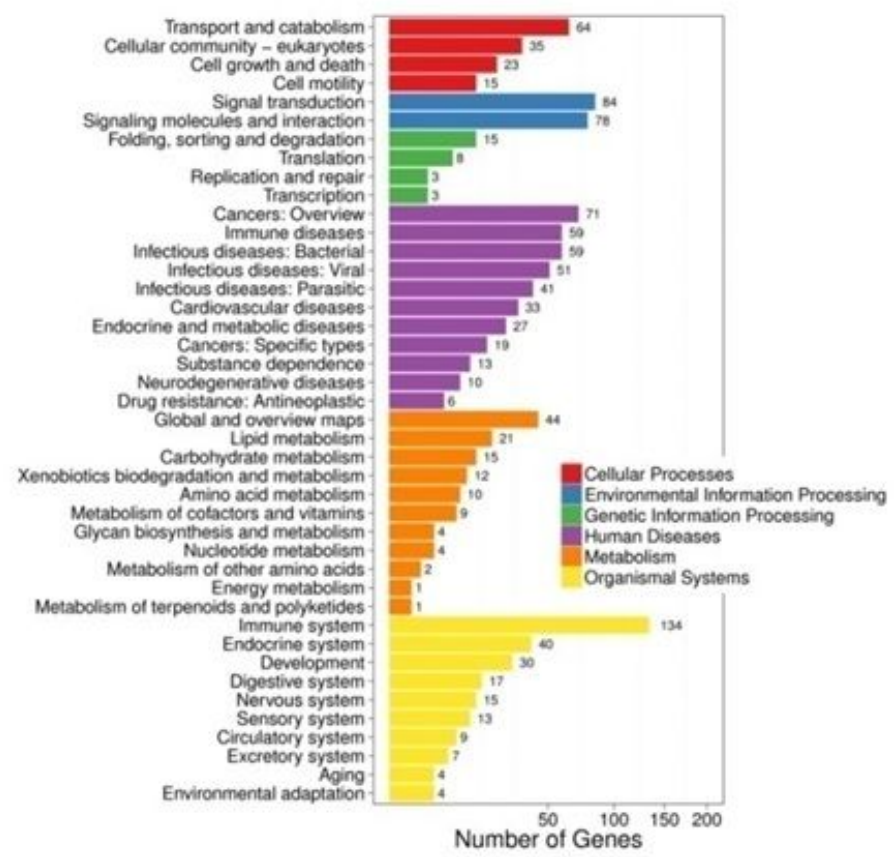

B

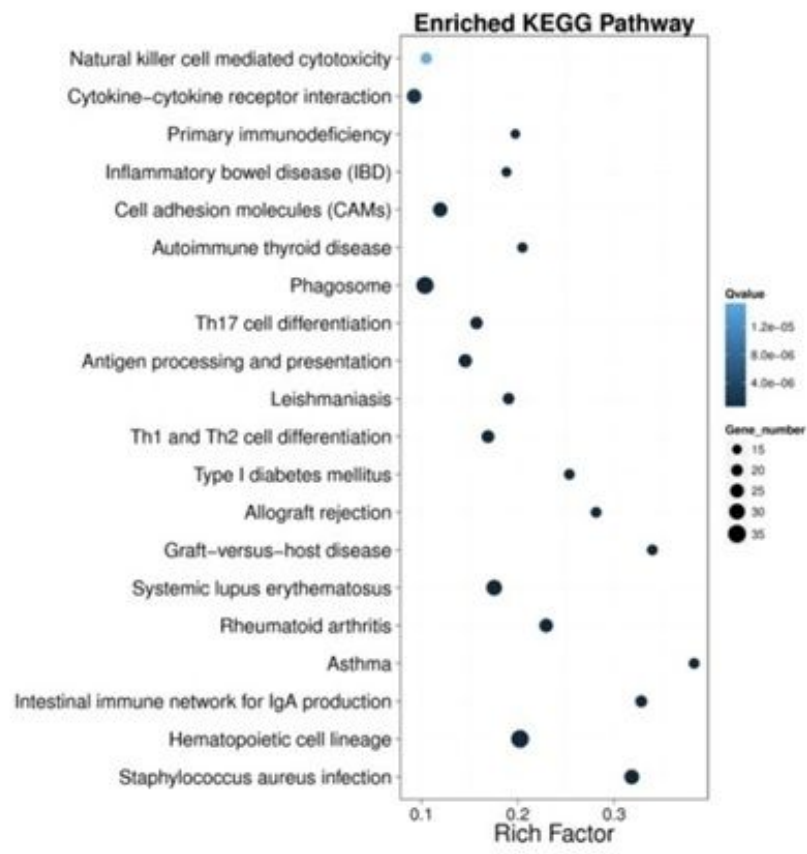

\section{Figure 2}

Pathway functional enrichment results of NT-VS-PT. DEGseq Pathway. (A) X axis represents number of DEG. Y axis represents functional classification of KEGG. There are seven branches for KEGG pathways: Cellular Processes, Environmental Information Processing, Genetic Information Processing, Human Disease (For animals only), Metabolism, Organismal Systems and Drug Development. (B) X axis represents enrichment factor. $Y$ axis represents pathway name. The color indicates the q-value (high: white, low: blue), the lower q-value indicates the more significant enrichment. Point size indicates DEG number (The bigger dots refer to larger amount). Rich Factor refers to the value of enrichment factor, which is the quotient of foreground value (the number of DEGs) and background value (total Gene amount). The larger the value, the more significant enrichment. 


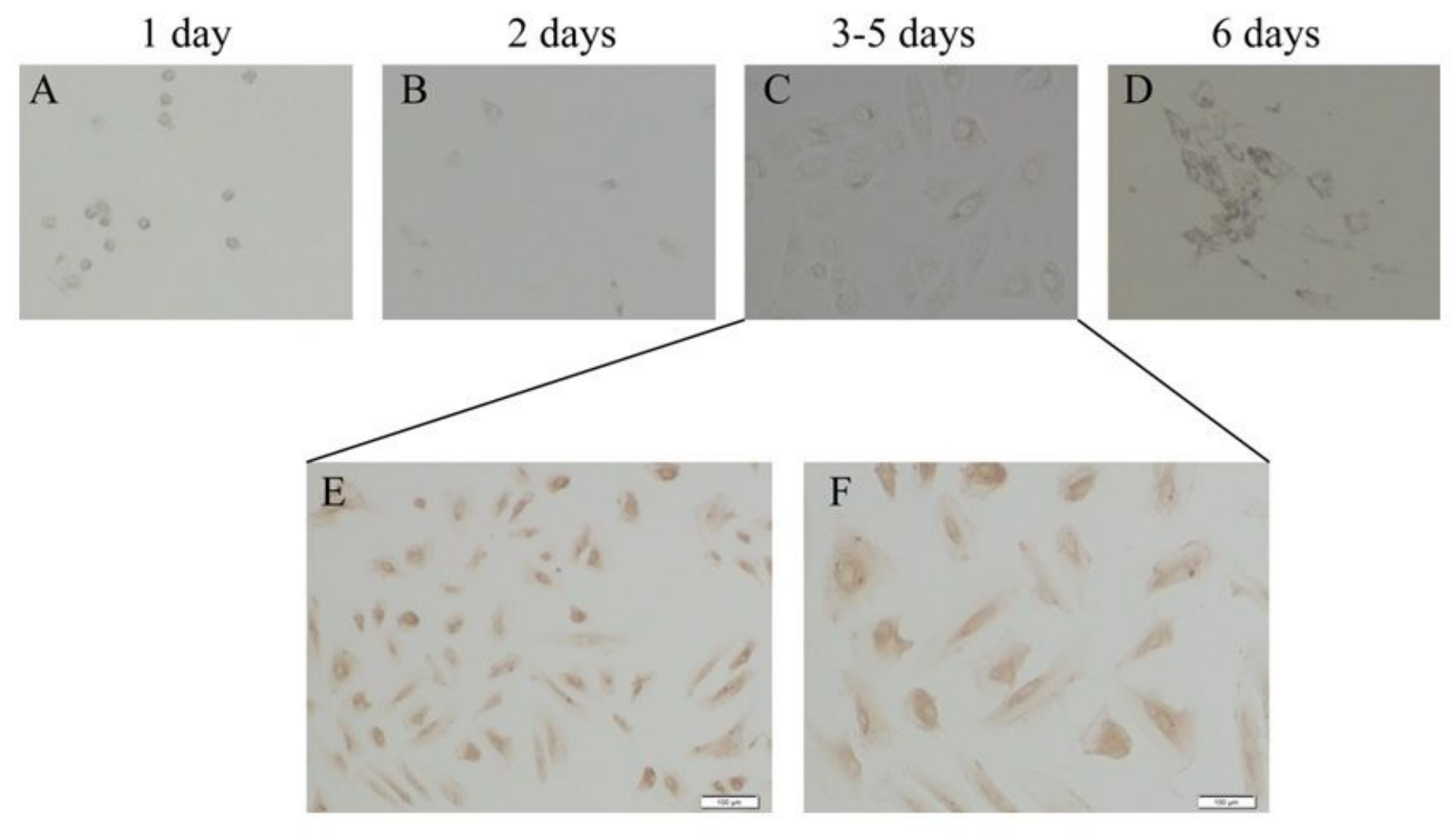

Figure 3. Culture and identification of ovarian GCs in vitro. (A)-(D) represent the

Figure 3

Culture and identification of ovarian GCs in vitro. (A)-(D) represent the situation of GCs cultured in vitro for 1-7 days. $(E)-(F)$ represent the IHC results of GCs staining with $\mathrm{FSH}$ antibody during 3-5 days. Scale bar $=100 \mu \mathrm{m}$ for $(E)$, Scale bar $=50 \mu \mathrm{m}$ for $(\mathrm{F})$.

A

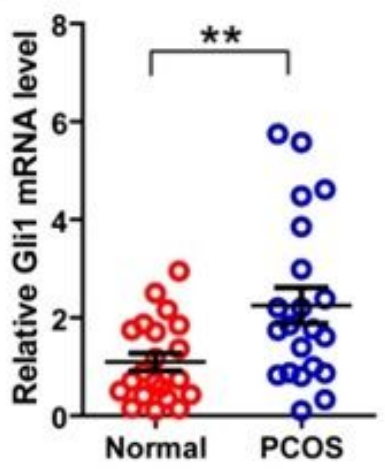

$\mathrm{B}$

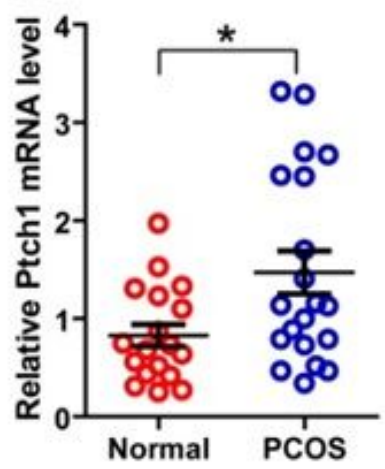

$\mathrm{C}$

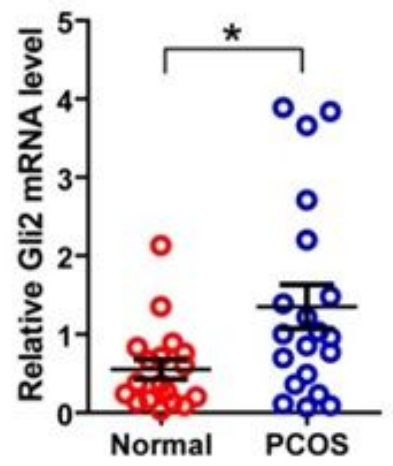

$\mathrm{D}$

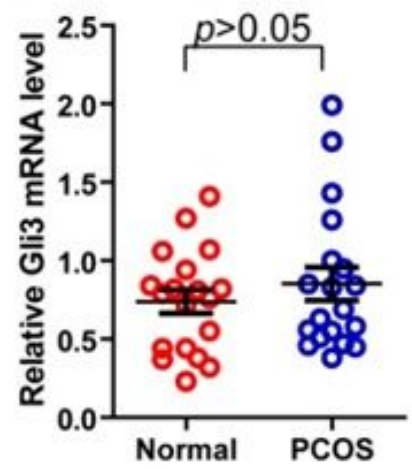

Figure 4 
Expression of Hh family members in GCs from PCOS group and control Group. $\llbracket A \rrbracket-\bigotimes D$ \represent Gli, Ptch1, Gli2 and Gli3 mRNA levels form GCs between normal and PCOS.. The red circle represents the normal group (Normal), and the blue circle represents the experimental group (PCOS). p-values were determined by Student's t-test, ${ }^{*} p<0.05,{ }^{*} \mathrm{p}<0.01$.

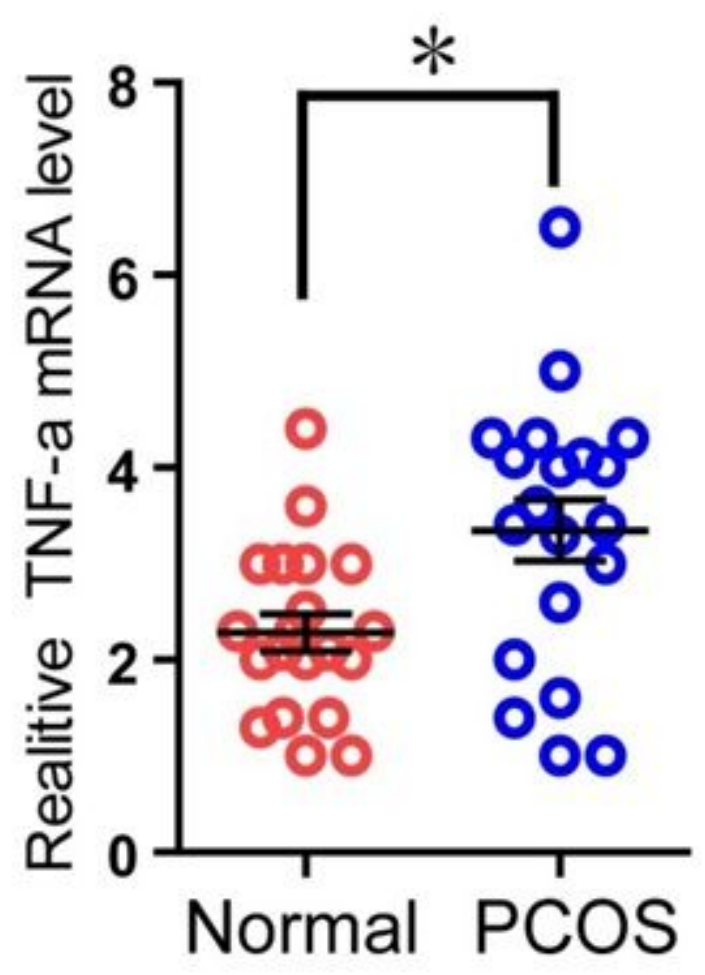

Figure 5

Comparison of TNF-a expression in granulosa cells of two groups. The relative difference of TNF-a mRNA expression results between the normal group (Normal) and the experimental group (PCOS). The red circle represents the normal group (Normal), and the blue circle represents the experimental group (PCOS). * $p<0.05$. 


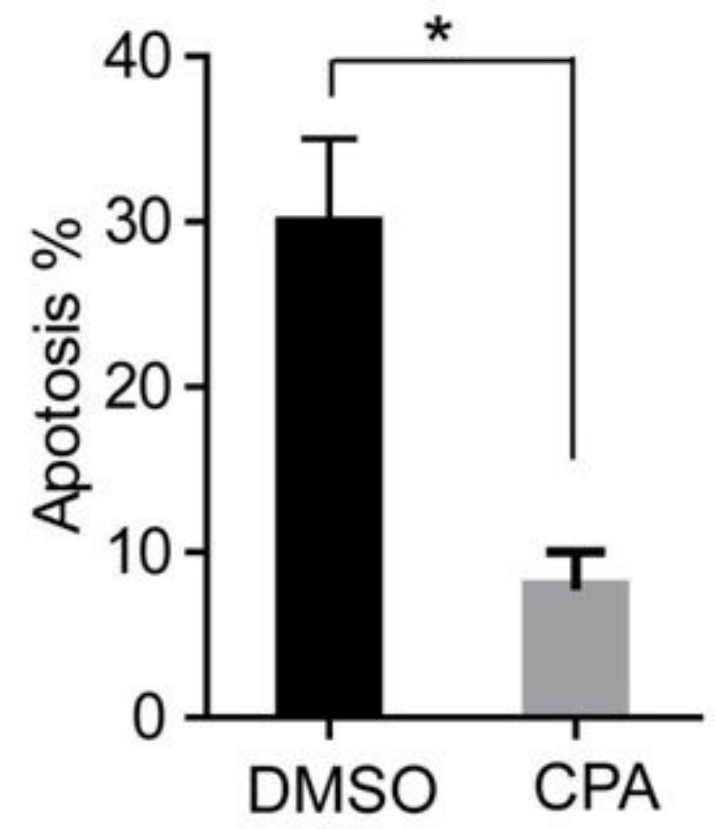

Figure 6

CPA treatment of PCOS ovarian granulosa cells to detect its apoptosis. PCOS cells were treated with KAAD-cyclopamine (CPA, 1um) 24 hours later to detect the apoptosis, and then compared with the control group (DMSO). * $p<0.05$. 